\title{
Thermally Activated Delayed Fluorescence from Locally Excited State: Theoretical Prediction and Experimental Validation
}

\author{
Xiaoxiao Xiao ${ }^{a \#}$, Shuai Lia\#, Hua Genga*, Yuai Duan" ${ }^{a \neq}$, Guo Wanga , Qian Peng ${ }^{b *}$, Zhigang Shuaic, \\ Hongbing $\mathrm{Fu}^{a}$, Yi Liao *
}

\begin{abstract}
As is well known, the thermally activated delayed fluorescence (TADF) is always generated from charge-transfer (CT) excited states in electron-donor $(D)$ - electron-acceptor $(A)$ systems. Here, a novel design strategy is proposed for realizing TADF from a locally excited (LE) state through controlling the intersystem crossing (ISC) and reverse intersystem crossing (RISC) processes between the LE singlet and higher triplet CT states. Based on the strategy, a boron difluoride derivative is theoretically predicted to emit TADF from the $L E$ state, whose radiative decay rate constant is much larger $k_{r}\left(S_{1} \rightarrow S_{0}\right)=1.12 \times 10^{8} s^{-1}$, two orders of magnitude larger than those of common TADF systems. And its lifetimes of the prompt and delayed fluorescence are experimentally validated to be $0.44 \mathrm{~ns}$ and $0.7 \mu \mathrm{s}$, respectively. This work is a breakthrough in the understanding of TADF and opens a new avenue for extending the TADF materials.
\end{abstract}

Thermally activated delayed fluorescence (TADF) materials can achieve $100 \%$ internal quantum efficiency (IQE) and attract great interest in optoelectronic and biological areas. Adachi's group proposed TADF mechanism with small energy separations $\Delta E\left(\mathrm{~S}_{1}-\mathrm{T}_{1}\right)$ between the lowest singlet $\mathrm{S}_{1}$ and triplet $\mathrm{T}_{1}$ state, and thus enhance intersystem crossing (ISC) and reverse intersystem crossing (RISC) probability. ${ }^{[1]}$ Based on quantum chemistry theory, the $\Delta E_{\mathrm{ST}}$ between the $\mathrm{S}_{1}$ and $\mathrm{T}_{1}$ states is proportional to the exchange energy, which is mainly determined by the overlap integral between the HOMO and LUMO. The larger HOMO and LUMO are separated, the weaker exchange interaction and then the smaller $\Delta E\left(\mathrm{~S}_{1}-\mathrm{T}_{1}\right)$ should be. Thus, the formation of chargetransfer (CT) excited states is the key to efficient ISC and RISC, which identifies a chemical template for the TADF materials: electron-donor (D) - electron-acceptor (A) frameworks. [2] Keeping this in mind, a variety of TADF materials are designed and synthesized with strong intramolecular CT, intermolecular CT, through-space/-bond CT and so on. ${ }^{[3]}$ In the D-A systems, the small $\Delta E\left(\mathrm{~S}_{1}-\mathrm{T}_{1}\right)$ is achieved owing to the strongly spaceseparated HOMO and LUMO, however the small transition dipole moments are inevitably generated, leading to small oscillator

[a] X. Xiao, S. Li, Dr. Y. Duan, Prof. H. Geng, Prof. G. Wang, Prof. H. Fu, Prof. Y. Liao

Department of Chemistry, Beijing Advanced Innovation Center for Imaging Theory and Technology

Capital Normal University

Beijing, 100048, China

E-mail: hgeng@cnu.edu.cn; yliao@cnu.edu.cn

[b] Prof. Q. Peng

Department Beijing National Laboratory for Molecular Sciences,

CAS Key Laboratory of Organic Solids

Institute of Chemistry, Chinese Academy of Sciences

Beijing, 100190, China

E-mail: qpeng@iccas.ac.cn

[c] Prof. Z. Shuai

Department of Chemistry,

Tsinghua University

Beijing 100083, China

[\#] These authors contributed equally to this work

Supporting information for this article is given via a link at the end of the document. strengths and radiative decay rate constants $\left(k_{r}\right) \cdot{ }_{\cdot}^{[4]}$ As a result, it is a dilemma to achieve small $\Delta E\left(\mathrm{~S}_{1}-\mathrm{T}_{1}\right)$ and large $k_{\mathrm{r}}$ simultaneously in TADF molecules. ${ }^{\text {[3a] }}$ Many efforts have been performed to balance this point. The J-aggregates with strong intermolecular CT not only improves the radiative decay rate but also significantly decreases the $\Delta E\left(\mathrm{~S}_{1}-\mathrm{T}_{1}\right)$ to induce high-efficient TADF even in the NIR region. ${ }^{[3 \mathrm{e}]}$ Ma's group has put forward hot exciton RISC mechanism with a hybridized local and charge transfer (HLCT) emissive state, ${ }^{[3 \mathrm{~d}, 5]}$ which accelerated the radiative decay process remarkably. However, achieving TADF materials with fast radiative rate or large emission cross section remains a great challenge.

All studies to date have been widely regarded the emissive $S_{1}$ with CT character as the precondition to achieve TADF materials. The locally excited (LE) state promising large transition dipole moment is completely excluded in designing TADF materials because of large $\Delta E\left(\mathrm{~S}_{1}-\mathrm{T}_{1}\right)$ of ca. $0.5-1.2 \mathrm{eV}$, ${ }^{[6]}$ impossibly realizing efficient RISC from $T_{1}$ to $S_{1}$ processes. However, the large $\Delta E\left(\mathrm{~S}_{1}-\mathrm{T}_{1}\right)$ probably provides a stage for the higher triplet states to play an important role in TADF. Here, we creatively modulate the ISC and RISC processes between the $S_{1}$ and higher triplet state to realize TADF from a complete LE state in the boron difluoride derivatives.

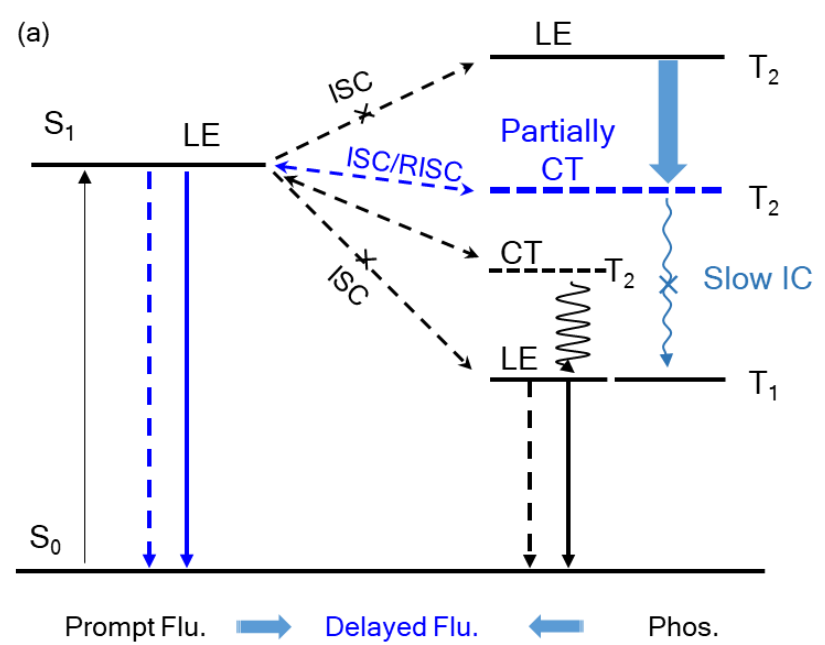

(b)

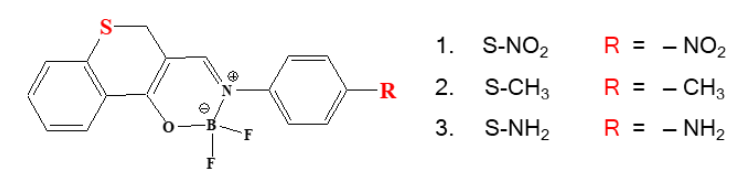

Scheme 1. (a) Design strategy in modulating among prompt fluorescence (Flu.) delayed fluorescence and phosphorescence (Phos.). (b) Molecular structures of 2,2-difluoro-3-phenyl-2H,5H-2/4,3/4-thiochromeno[3,4-e][1,3,2] oxazaborinine derivatives (S-R). 
The derivatives based on the 2,2-difluoro-3-phenyl-2H,5H2|4,3|4-thiochromeno[3,4-e][1,3,2] oxazaborinine exhibit wonderful light emitting properties from fluorescence, TADF to room-temperature phosphorescence. ${ }^{[7]}$ Moreover, the calculated results suggest the potentially tuneable energy gaps between the $S_{1}$ (or $T_{1}$ ) and $T_{2}{ }^{[8]}$ Triggered from this, we theoretically propose a novel design strategy to realize TADF from a complete LE state through controlling the occurrence of ISC and RISC process between $S_{1}$ and $T_{2}$, as shown in Scheme 1. The large energy gap between $S_{1}$ and $T_{1}$ remains almost unchanged. Through tuning the proportion of LE/CT component for $T_{2}$, the $\triangle E\left(S_{1}-T_{2}\right)$ is minimized for efficient RISC, while the $\Delta E\left(T_{2}-T_{1}\right)$ is maximized for suppressing the nonradiative decay to harvest $T_{1}$. More importantly, the spin-orbit coupling (SOC) between $S_{1}$ and $T_{2}$ is very large owing to the participation of lone pair electrons of sulfur atom, ${ }^{\left[{ }^{9]}\right.}$ which largely enhances the mutual conversion $S_{1} \leftrightarrow T_{2}$ processes. Based on the strategy above, by changing a small substituent to the backbone, we theoretically predicted $\mathrm{S}-\mathrm{CH}_{3}$ emitting TADF from the LE state and its lifetimes of the prompt and delayed fluorescence are experimentally validated to be 0.44 ns and $0.7 \mu \mathrm{s}$, respectively. This work is a breakthrough in the understanding of TADF and opens a new avenue for extending the TADF materials, especially for the organic laser TADF materials which need a large emission cross section. ${ }^{[10]}$

The (E)3-((4-nitrophenyl)imino)methyl)-2H-thiochroman-4olate-BF2 $\left(\mathrm{S}-\mathrm{NO}_{2}\right)$ exhibits excellent room temperature phosphorescence in solution $[\underline{[b]}$ via efficient ISC process from the $\mathrm{S}_{1}$ to $\mathrm{T}_{2}$ states. ${ }^{[8]}$ When substituting the electron-withdrawing $-\mathrm{NO}_{2}$ for a neutral methyl group $\left(-\mathrm{CH}_{3}\right)$ or an electron-detonating group $\left(-\mathrm{NH}_{2}\right)$ into backbone, we obtain 2,2-difluoro-3-(p-tolyl)- $2 \mathrm{H}, 5 \mathrm{H}$ $2 \lambda 4,3 \lambda 4$-thiochromeno[3,4-e][1,3,2]oxazaborinine, $\left(\mathrm{S}-\mathrm{CH}_{3}\right)$ and 4 (2,2-difluoro-2H,5H-2/4,314-thiochromeno e][1,3,2]oxazaborinin-3-yl)aniline $\left(\mathrm{S}-\mathrm{NH}_{2}\right)$ systems. We firstly theoretically calculated the geometrical and electronic structures of the three compounds at (TD) DFT level, and quantitatively predicted their photophyscial properties using the thermal vibration correlation function rate theory.

Table 1. The optimized geometric structures of the low-lying singlet and triplet excited states for the investigated systems.

\begin{tabular}{c|ccccccccc}
\hline \multirow{2}{*}{ Systems } & & & & & & & & & \\
\cline { 2 - 9 } & $\mathrm{S}_{0}$ & $\mathrm{~S}_{1}$ & $\mathrm{~T}_{1}$ & $\mathrm{~T}_{2}$ & $\mathrm{~S}_{0}$ & $\mathrm{~S}_{1}$ & $\mathrm{~T}_{1}$ & $\mathrm{~T}_{2}$ \\
\hline $\mathrm{S}_{\mathrm{N}} \mathrm{NO}_{2}$ & 38.57 & 26.72 & 25.06 & 26.77 & 1.420 & 1.394 & 1.392 & 1.392 \\
$\mathrm{~S}-\mathrm{NH}_{2}$ & 42.48 & 23.51 & 19.43 & 34.26 & 1.430 & 1.386 & 1.368 & 1.412 \\
\hline
\end{tabular}

The main optimized geometry parameters are shown in Table 1 for the three systems at the ground state $S_{0}$ and excited $S_{1}, T_{1}$ and $T_{2}$ states. The three molecules in all the involved electronic states are non-planar with the twisted dihedral angle $\theta$ between B-group and phenyl-group (as marked in Table 1) in the range of $16^{\circ} \sim 43^{\circ}$. More interestingly, from $\mathrm{S}-\mathrm{NO}_{2}$ to $\mathrm{S}-\mathrm{NH}_{2}$, the geometric structures have little change (less than $7^{\circ}$ ) for the $S_{0}$ and $S_{1}$ states, while there are large variations for the $T_{1}$ and $T_{2}$ states. The dihedral angle $\theta$ decreases by $8.87^{\circ}$ in the $T_{1}$ state, while increases up to $12.93^{\circ}$ in $T_{2}$ state. The main bond lengths also display the same change trend. These indicate that the substitutions with different electronegativity have a large effect on the triplet state, especially the $T_{2}$ state, which perfectly meets our expectations.

As expected, the energy gap between $T_{1}$ and $T_{2}$ states is successfully modulated from $0.37 \mathrm{eV}$ of S-NO $\mathrm{NO}_{2}, 0.63 \mathrm{eV}$ of S-CH to $0.93 \mathrm{eV}$ of $\mathrm{S}-\mathrm{NH}_{2}$, as can be seen in Figure 1a. Relative to the $S_{1}$, the $T_{2}$ is raised lower than $S_{1}$ for $S-N_{2}$, very close to $S_{1}$ for $S$ $\mathrm{CH}_{3}$, and higher than $\mathrm{S}_{1}$ for $\mathrm{S}-\mathrm{NH}_{2}$. In addition, the SOCs $(\xi)$ between the singlet and triplet states of the three molecules are much stronger compared with common metal-free organic molecules owing to the participation of lone pair electrons ( $n$ orbital) of sulfur atom (see Table $\mathrm{S} 1$ ). Especially, the $\xi\left(\mathrm{S}_{1}, \mathrm{~T}_{1}\right)$ and $\xi\left(\mathrm{S}_{1}, \mathrm{~T}_{2}\right)$ are up to $17.0-20.0 \mathrm{~cm}^{-1}$ for $\mathrm{S}-\mathrm{NO}_{2}$ and $\mathrm{S}-\mathrm{CH}_{3}$, which greatly boost the ISC/RISC between singlet and triplet states.
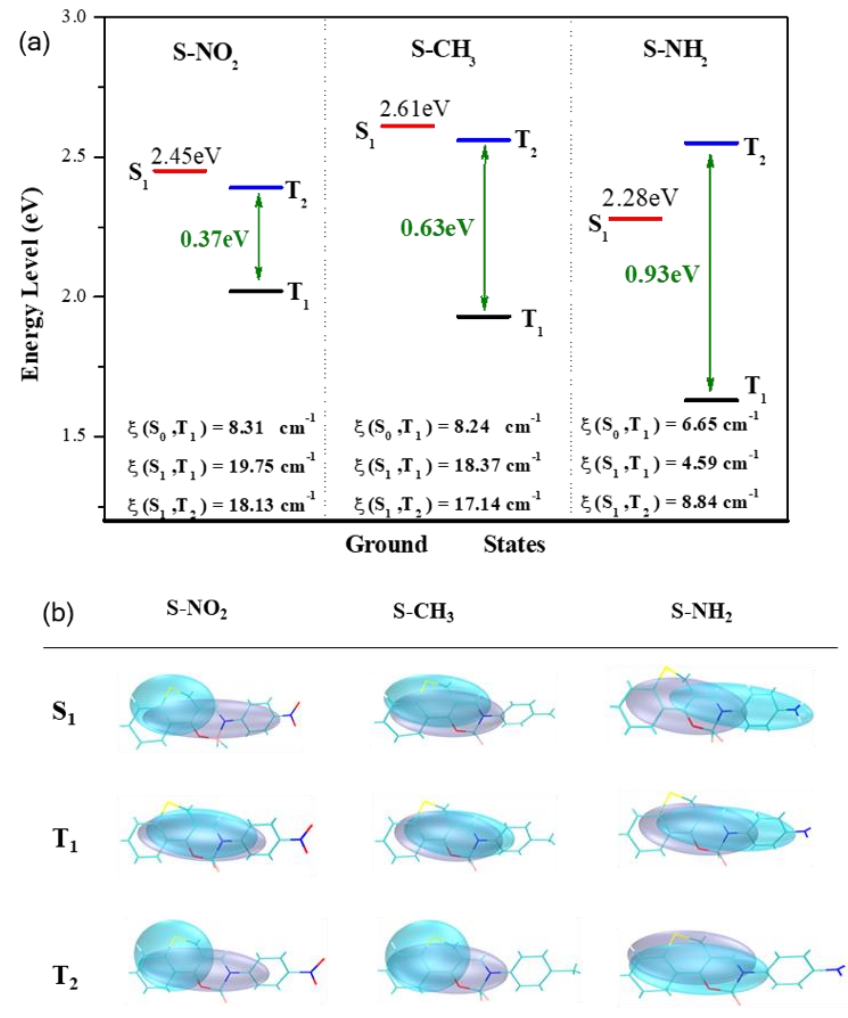

Figure 1. (a) The energy level diagram and spin-orbit couplings constants $(\xi)$ between singlet and triplet states, and (b) Hole-electron analysis with Cele (cyan) and Chole (iceblue) image (isovalue $=0.001$ ) of the three compounds in DCM based on the optimized geometries.

These resultant energy levels and electronic properties tell us that (i) for $\mathrm{S}-\mathrm{NO}_{2}$, the small $\Delta E\left(\mathrm{~S}_{1}-\mathrm{T}_{2}\right)$ and large $\xi\left(\mathrm{S}_{1}, \mathrm{~T}_{2}\right)$ bring up an efficient ISC process from the $S_{1}$ to $T_{2}$ state, and then experience fast internal conversion (IC) from $T_{2}$ to $T_{1}$ owing to small $\Delta E\left(\mathrm{~T}_{1}-\mathrm{T}_{2}\right)$ of $0.37 \mathrm{eV}$, which urges the occurrence of the RTP as observed in experiment; [7b] (ii) for $\mathrm{S}-\mathrm{NH}_{2}$, the ISC between singlet and triplet states is impossible for the exorbitant energy of $\mathrm{T}_{2}$ and 


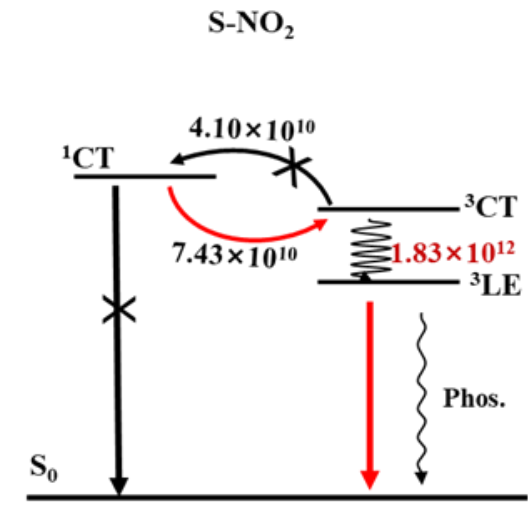

RTP

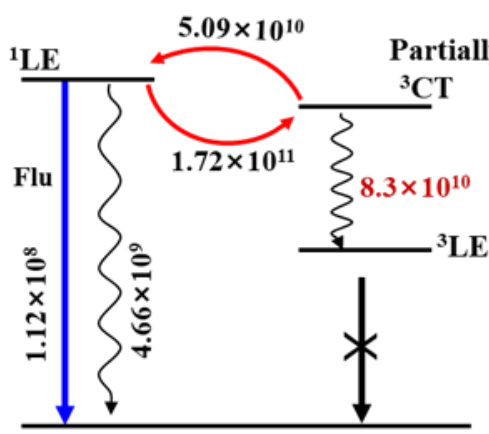

TADF
$\mathrm{S}-\mathrm{NH}_{2}$

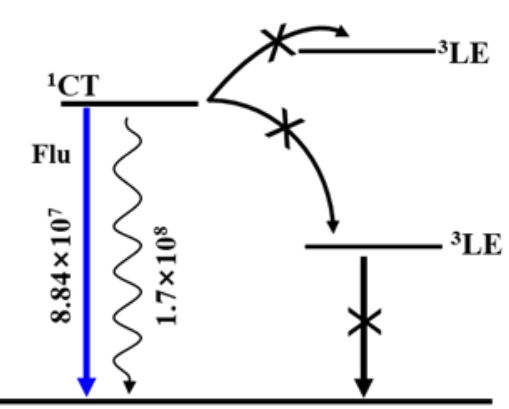

Prompt Fl.

Figure 2. Computed related rate constant for photophysical processes.

too large $\Delta E\left(\mathrm{~S}_{1}-\mathrm{T}_{1}\right)$ of $0.65 \mathrm{eV}$, and there are not any predicted TADF or RTP; (iii) for S- $\mathrm{CH}_{3}$, the extremely narrow energy gap with $\Delta E\left(\mathrm{~S}_{1}-\mathrm{T}_{2}\right)=0.05 \mathrm{eV}$ and large $\xi\left(\mathrm{S}_{1}, \mathrm{~T}_{2}\right)$ assure to open the ISC and RISC pathways between $S_{1}$ and $T_{2}$. Moreover, the $\Delta E\left(T_{1}-T_{2}\right)$ is increased to $0.63 \mathrm{eV}$ from the $0.37 \mathrm{eV}$ of $\mathrm{S}-\mathrm{NO}_{2}$, which sharply slows down the IC process from $T_{2}$ to $T_{1}$. Therefore, TADF would be observed if the RISC rate is comparable to the IC rate from $T_{2}$ to $\mathrm{T}_{1}$.

To figure out the nature of the excited states, we further performed the hole-electron analysis (Figure 1b) and quantitatively calculated the CT amount (Figure S2) by the MULTIWFN program ${ }^{[11]}$ based on the molecular configuration information of the excited states in Table S2 and Figure S1. It is easily seen that the $\mathrm{S}_{1}$ state of $\mathrm{S}-\mathrm{NO}_{2}$ exhibits obvious CT character (48.9\%) owing to the strong electron-withdrawing ability of $-\mathrm{NO}_{2}$, and its transition electric dipole moment $(\mu)$ is 7.09 Debye The $\mathrm{T}_{2}$ also shows remarkable CT character $(36.1 \%)$, which is $0.37 \mathrm{eV}$ higher than $\mathrm{T}_{1}$ with $\mathrm{LE}$ character. When introducing the neutral methyl group $\left(-\mathrm{CH}_{3}\right)$ in backbone as $\mathrm{S}-\mathrm{CH}_{3}$, the $\mathrm{S}_{1}$ state mainly originates from locally excited $\Pi \rightarrow \pi^{*}$ transition, in which the excitation energy is promoted and the $\mu$ is amplified up to 8.68 Debye relative to those of $\mathrm{S}-\mathrm{NO}_{2}$ (the current $\mu$ is much higher than those of most TADF molecules $\left.{ }^{[12]}\right)$. More importantly, the CT component of the $T_{2}$ state decreases to some extent, which increases the energy level of the $T_{2}$ to be very close to the $S_{1}$ and far away from the LE $T_{1}$. When going to the electron-donating ($\mathrm{NH}_{2}$ ) group, the $\mathrm{S}_{1}$ and $\mathrm{T}_{1}$ states both changed to have some CT features, remaining large energy difference of $0.65 \mathrm{eV}$. While the $\mathrm{T}_{2}$ changes from CT to LE and its energy is increased to be much higher than that of the $S_{1}$. Therefore, the modulation of energy level structure in Scheme 1 has been perfectly realized.

To accurately work out the competitive results of the dynamic processes, including ISC, RISC and IC, and fluorescence, we quantitatively evaluated the rate constants of the processes at $298 \mathrm{~K}$ by using the thermal vibration correlation function (TVCF) formalisms in MOMAP program ${ }^{[13]}$ and gave them in Figure 2 and Table S3. For S-NO $\mathrm{NO}_{2}$, the radiative decay rate constant $k_{\mathrm{r}}\left(\mathrm{S}_{1} \rightarrow \mathrm{S}_{0}\right)$ is $4.57 \times 10^{7} \mathrm{~s}^{-1}$, which is a typical value of a hybrid LE and CT emission state. $\left[\frac{[3 d, 5]}{3}\right.$ The nonradiative decay rate from $S_{1}$ to $S_{0}$ $k_{\mathrm{IC}}\left(\mathrm{S}_{1} \rightarrow \mathrm{S}_{0}\right)$ is $3.12 \times 10^{10} \mathrm{~S}^{-1}$, which is very fast owing to very large geometry modifications between the $S_{1}$ and $S_{0}$ states, as list in Table 1. The ISC from $S_{1}$ to $T_{2}$ and the IC from $T_{2}$ to $T_{1}$ are both extremely fast with $k_{\mathrm{ISC}}\left(\mathrm{S}_{1} \rightarrow \mathrm{T}_{2}\right)=7.43 \times 10^{10} \mathrm{~s}^{-1}$ and $k_{\mathrm{IC}}\left(\mathrm{T}_{2} \rightarrow \mathrm{T}_{1}\right)$ $=1.83 \times 10^{12} \mathrm{~s}^{-1}$, and the RISC rate constant from $T_{2}$ to $S_{1}$ of $4.10 \times 10^{10} \mathrm{~s}^{-1}$ is two orders of magnitude smaller than the $k_{\mathrm{IC}}\left(\mathrm{T}_{2} \rightarrow \mathrm{T}_{1}\right)$, which greatly harvest the population of the $T_{1}$ state.
Therefore, there is no clear fluorescence for $\mathrm{S}-\mathrm{NO}_{2}$ as observed in experiment. ${ }^{\left[{ }^{[b]}\right]}$ Moreover, the lifetime of the room temperature phosphorescence of $\mathrm{S}-\mathrm{NO}_{2}$ is calculated to be $6.13 \mu \mathrm{s}$, which is in good agreement with the experimental value $8.3 \pm 0.1 \mu \mathrm{s}$ [b] $^{[7 \mathrm{~b}}$ For $\mathrm{S}-\mathrm{NH}_{2}$, there is only conventional fluorescence to be predicted with the radiative and nonradiative decay rates of $8.84 \times 10^{7} \mathrm{~s}^{-1}$ and $1.7 \times 10^{8} \mathrm{~s}^{-1}$.

More excitingly, it is safely predicted TADF can happen from the LE state for $\mathrm{S}-\mathrm{CH}_{3}$ because (i) its radiative decay rate constant $k_{r}\left(S_{1} \rightarrow S_{0}\right)$ is $1.12 \times 10^{8} \mathrm{~s}^{-1}$, which is a typical value of the LE state and two orders of magnitude larger than those of common TADF

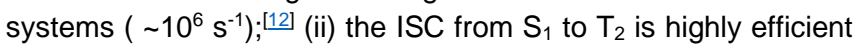
with $k_{I S C}\left(S_{1} \rightarrow T_{2}\right)=1.72 \times 10^{11} \mathrm{~s}^{-1}$, however the $k_{I C}\left(T_{2} \rightarrow T_{1}\right)$ of $8.3 \times 10^{10} \mathrm{~s}^{-1}$ and $k_{\mathrm{RISC}}\left(\mathrm{T}_{2} \rightarrow \mathrm{S}_{1}\right)$ of $5.09 \times 10^{10} \mathrm{~s}^{-1}$ is quite comparable which result in the coexistence of the $T_{1}$ and $S_{1}$ states; (iii) from $\mathrm{T}_{1}$ to $\mathrm{S}_{0}$ state, the nonradiative decay rate $k_{\mathrm{NR}}\left(\mathrm{T}_{1} \rightarrow \mathrm{S}_{0}\right)$ is far larger than the radiative decay rate $k_{P}\left(T_{1} \rightarrow S_{0}\right)$, leading to no phosphorescence at room temperature; and (iv) from $S_{1}$ to $S_{0}$, the radiative and nonradiative decay processes are able to be compared, generating prompt fluorescence and TADF. In addition, it can be found that owing to large SOC, minor energy gap $(0.05$ $\mathrm{eV})$ and small reorganization energy $\left(0.12 \mathrm{eV}\right.$ or $\left.978.83 \mathrm{~cm}^{-1}\right)$, the ISC and RISC rates of the $S_{1} \leftrightarrow T_{2}$ are much larger than those of traditional TADF molecules from $S_{1} \leftrightarrow T_{1},{ }^{[12]}$ which portends the shorter lifetime of TADF of $\mathrm{S}-\mathrm{CH}_{3}$. Thus far, the TADF from the LE state is quantitatively predicted from a dynamics rate point of view.

To confirm the TADF behaviors from the LE state in $\mathrm{S}-\mathrm{CH}_{3}$, we facilely synthesized $\mathrm{S}-\mathrm{CH}_{3}$ through two-step reactions in $\mathrm{SI}^{\dagger}$, characterized by ${ }^{1} \mathrm{H} \mathrm{NMR}$ in $\mathrm{SI}^{\dagger}$, and performed the steady-state and transient photoluminescence $(\mathrm{PL})$ spectra at $298 \mathrm{~K}$ and $77 \mathrm{~K}$ in DCM solution shown in Figure 3 and Figure S6. For comparison, the calculated absorption spectrum of the $S_{0} \rightarrow S_{1}$ and fluorescence spectrum at $298 \mathrm{~K}$ are plotted in Figure $3 \mathrm{a}$. From Figure $3 a$, it is easily seen that the experimental spectra are in good accordance with the theoretical predictions. The 0-0 peak positions of $S_{0} \leftrightarrow S_{1}$ and $S_{0} \leftrightarrow T_{1}$ is theoretically calculated to be 2.51 and $1.84 \mathrm{eV}$, which are very close to the experimental counterparts 2.56 and $1.96 \mathrm{eV}$. Figure $3 \mathrm{a}$ shows the prompt component (magenta line) integrated over the nanosecond time range and delayed one (green line) recorded upon time gating (after $0.1 \mu \mathrm{s}$ ), as well as the steady-state PL spectrum (black line) for comparison. It is apparent that the prompt and delayed PL spectra are very similar to steady-state PL spectrum, which indicates that they originate from the same excited singlet state. Figure $3 \mathrm{~b}$ plots the transient $\mathrm{PL}$ decay curves at $298 \mathrm{~K}$ and $77 \mathrm{~K}$ 
There are obvious two-component decays, a fast decay with a lifetime of $0.44 \pm 0.002 \mathrm{~ns}$ and a slow decay with a lifetime of 0.7 $\pm 0.08 \mu$ s (black line) in air at $298 \mathrm{~K}$.
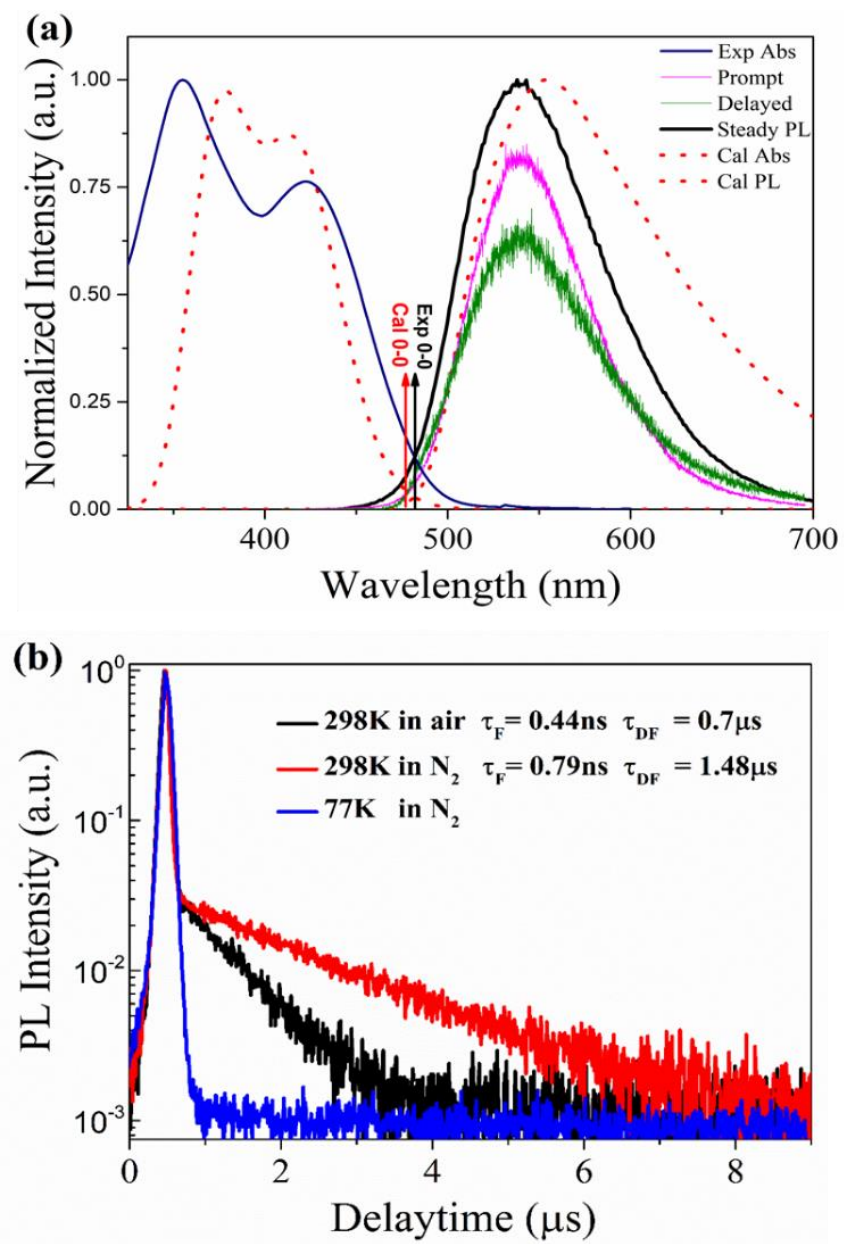

Figure 3. (a) Steady-state absorption (navy blue line) and transient steady $\mathrm{PL}$ spectra (black line) at room temperature: prompt component (magenta line), delayed component (green line) of $\mathrm{S}-\mathrm{CH}_{3}$ in DCM solutions (ca. $1 \times 10-5 \mathrm{M}$ ). The calculated absorption and PL spectra are shown as red dot line. (b) The PL decay of $\mathrm{S}-\mathrm{CH}_{3}$ in $\mathrm{DCM}$ at $298 \mathrm{~K}$ and $77 \mathrm{~K}$ (blue line). Black and red lines show the profiles before and after the deoxygenation, respectively.

Table 2. Photophysical parameters of $\mathrm{S}-\mathrm{CH}_{3}$ compound in DCM solution.

\begin{tabular}{cccc}
\hline $\mathrm{S}-\mathrm{CH}_{3}$ & $\lambda_{\mathrm{PL}}[\mathrm{nm}]$ & $k_{\mathrm{r}}\left[\mathrm{s}^{-1}\right]$ & $\tau_{\mathrm{PF}}[\mathrm{ns}]$ \\
\hline $\mathrm{Exp}$ & 549 & $1.16 \times 10^{8}$ & 0.4 \\
\hline $\mathrm{Cal}$ & 554 & $1.12 \times 10^{8}$ & 0.21 \\
\hline
\end{tabular}

Excitingly, upon deoxygenated, the lifetime of prompt fluorescence is increased to $0.79 \pm 0.4 \mathrm{~ns}$ and the delayed one is extended to $1.48 \pm 0.058 \mu \mathrm{s}$ (red line), which is the typical feature of TADF. More importantly, it is found that the delayed emission component vanishes at $77 \mathrm{~K}$ (blue line), which is also the unique nature of TADF. The prompt fluorescence rate can be inferred from prompt fluorescence efficiency and corresponding lifetime to be $1.16 \times 10^{8} \mathrm{~s}^{-1}$, which is close to the theoretical radiative decay rate constant $1.12 \times 10^{8} \mathrm{~s}^{-1}$, As list in Table 2. All of this experimental results completely validate the theoretical prediction that the TADF can happen from the LE state in molecules like S$\mathrm{CH}_{3}$, which is extraordinarily important for extending the TADF systems. Especially, it would open the door to develop organic laser materials based on TADF because the large emission cross section is a very important requirement for an organic laser material.

To summarize, we proposed a novel design strategy to realize TADF based on a complete locally-excited-type molecule from first principles, in which reverse intersystem crossing (RISC) channel is opened through tuning the LE $S_{1}$ state and higher triplet state. Based on the strategy, through modifying different electronegativity substituents to boron difluoride derivative, the $T_{2}$ energy level is tuned via the proportion of LE/CT component while the large energy gap between $S_{1}$ and $T_{1}$ remains little change. The $\Delta E\left(\mathrm{~S}_{1}-\mathrm{T}_{2}\right)$ is minimized for efficient RISC, while the $\Delta E\left(\mathrm{~T}_{2-}\right.$ $T_{1}$ ) is maximized for suppressing the nonradiative decay. Additionally, the spin-orbit coupling (SOC) between $\mathrm{S}_{1}$ and $\mathrm{T}_{2}$ in the boron difluoride derivative is so large due to the participation of lone pair electrons of sulfur atom, which largely enhances the mutual conversion $S_{1} \leftrightarrow T_{2}$ processes. As expected, the boron difluoride derivative $\mathrm{S}-\mathrm{CH}_{3}$ is predicted to emit TADF from the locally excited state from the quantum chemistry and dynamics rate constants calculations. Its radiative decay rate constant is much larger $k_{r}\left(S_{1} \rightarrow S_{0}\right)=1.12 \times 10^{8} \mathrm{~s}^{-1}$, which is two orders of magnitude larger than those of common TADF systems. Moreover, its TADF property is experimentally validated through transient photoluminescence spectra at $298 \mathrm{~K}$ and $77 \mathrm{~K}$ in DCM solution, whose prompt and delayed fluorescence lifetimes are measured to be $0.44 \mathrm{~ns}$ and $0.7 \mu \mathrm{s}$, respectively. This work breaks through the traditional wisdom "the charge-transfer excited state is necessary prerequisite to the occurrence of TADF in organic molecules". Thus, our work provides a novel mechanism of TADF and open an avenue to extend the TADF materials, especially the organic laser emitters based on TADF.

\section{Experimental and Computational details}

Theoretical calculations were carried out through density functional theory (DFT) and time-dependent DFT (TD-DFT) combined with Polarizable Continuum Model (PCM), all the molecules were modeled in dichloromethane solution. Geometrical and electronic structures are performed at the B3LYP/6-31G* level in Gaussian 09 program package. ${ }^{14]}$ The spin-orbit coupling (SOC) constants were estimated by employing the Breit-Pauli spin-orbit Hamiltonian with an effective charge approximation implemented in the PySOC code. ${ }^{[15]}$ The derivative coupling between $T_{2}$ and $T_{1}$ are evaluated through pseudowavefunction ansatz method implemented in Qchem. ${ }^{[16]}$ The radiative decay $\left(k_{\mathrm{r}}, k_{\mathrm{P}}\right)$ and non-radiative decay $\left(k_{\mathrm{ISC}}, k_{\mathrm{RISC}}, k_{\mathrm{IC}}\right.$, $\left.k_{N R}\right)$ are calculated though thermal vibration correlation function rate theory implemented in MOMAP (Molecular Materials Property Prediction Package) program. ${ }^{[13]}$

All the chemicals and reagents were purchased from commercial sources and used as received without further purification. All reactions involving air-sensitive reagents or intermediates were carried out under argon atmosphere. If not indicated otherwise, all photophysical measurements were performed in dried solvents. The molecule synthesized was purified by column chromatography and recrystallization from dichloromethane and hexane for two times, and fully characterized by ${ }^{1} \mathrm{H}$ NMR, ${ }^{13} \mathrm{C}$ NMR. ${ }^{1} \mathrm{H}$ NMR $(400 \mathrm{MHz})$ and ${ }^{13} \mathrm{C}$ NMR $(151 \mathrm{MHz})$ spectra were recorded in deuterated solvents on a Bruker ADVANCE 400 NMR Spectrometer. Tetramethylsilane (TMS) was used as the internal standard. The UV-visible absorption spectra were measured on a Shimadzu UV-3600 spectrometer with a slit width of $1 \mathrm{~nm}$. The fluorescence emission spectroscopy, temperature dependent photoluminescence 
spectra were measured on a Horiba FluoroMax-4-NIR spectrophotometers.

\section{Acknowledgements}

This work is supported by National Key R\&D Program of China (2017YFA0204502 and 2017YFA0204700) and the National Natural Science Foundation of China (21973099 and 21673247), Beijing Municipal natural science Foundation (2182012 and 2192013) and Capacity Building for Sci-Tech InnovationFundamental Scientific Research Funds (19530012018 and 19530050138), and the Strategic Priority Research Program of the Chinese Academy of Sciences (XDB12020200).

\section{Conflict of interest}

The authors declare no conflict of interest.

Keywords: locally excited states $\bullet$ thermally active delayed fluorescence $\bullet$ charge transfer $\bullet$ triplet states

\section{References}

[1] H. Uoyama, K. Goushi, K. Shizu, H. Nomura, C. Adachi, Nature 2012, 492, 234-+.

[2] a) C. Adachi, Jpn J Appl Phys 2014, 53, 060101. b) Y. Geng, A. D'Aleo, K. Inada, L. S. Cui, J. U. Kim, H. Nakanotani, C. Adachi, Angew Chem Int Ed Engl 2017, 56, 16536-16540.

[3] a) D.-H. Kim, A. D'Aléo, X.-K. Chen, A. D. S. Sandanayaka, D. Yao, L. Zhao, T. Komino, E. Zaborova, G. Canard, Y. Tsuchiya, E. Choi, J. W. Wu, F. Fages, J.-L. Brédas, J.-C. Ribierre, C. Adachi, Nat Photonics 2018, 12, 98-104; b) L. J. Sun, W. J. Hua, Y. Liu, G. J. Tian, M. X. Chen, M. X. Chen, F. X. Yang, S. F. Wang, X. T. Zhang, Y. Luo, W. P. Hu, Angew Chem Int Edit 2019; c) Y. K. Wang, C. C. Huang, H. Ye, C. Zhong A. Khan, S. Y. Yang, M. K. Fung, Z. Q. Jiang, C. Adachi, L. S. Liao, Adv Opt Mater 2019, 8, 1901150; d) W. J. Li, Y. Y. Pan, L. Yao, H. C. Liu, S. T. Zhang, C. Wang, F. Z. Shen, P. Lu, B. Yang, Y. G. Ma, Adv Opt Mater 2014, 2, 892-901; e) J. Xue, Q. X. Liang, R. Wang, J. Y. Hou, W. Q. Li, Q. Peng, Z. G. Shuai, J. Qiao, Adv Mater 2019, 31; f) J. Huang, H. Nie, J. Zeng, Z. Zhuang, S. Gan, Y. Cai, J. Guo, S. J. Su, Z. Zhao, B. Z. Tang, Angew Chem Int Ed Eng/ 2017, 56, 12971-12976; g) H. Tsujimoto, D. G. Ha, G. Markopoulos, H. S. Chae, M. A. Baldo, T. M. Swager, J Am Chem Soc 2017, 139, 4894-4900; h) J. Hu, Q. Li, X. Wang, S. Shao, L. Wang X. Jing, F. Wang, Angew Chem Int Ed Engl 2019, 58, 8405-8409; i) S. Shao, J. Hu, X. Wang, L. Wang, X. Jing, F. Wang, J Am Chem Soc 2017, 139, 17739-17742; j) J. Sun, J. Zhang, Q. Liang, Y. Wei, C. Duan, C. Han, H. Xu, Adv Funct Mater 2019, 30, 1908568; k) X. Zheng, R. Huang, C. Zhong, G. Xie, W. Ning, M. Huang, F. Ni, F. B. Dias, C. Yang, Adv Sci (Weinh) 2020, 7, 1902087.

[4] a) X. K. Chen, Y. Tsuchiya, Y. Ishikawa, C. Zhong, C. Adachi, J. L. Bredas, Adv Mater 2017, 29; b) M. Y. Wong, E. Zysman-Colman, Adv Mater 2017, 29.

[5] a) L. F. Chen, S. T. Zhang, H. Li, R. F. Chen, L. Jin, K. Yuan, H. H. Li, P. Lu, B. Yang, W. Huang, J Phys Chem Lett 2018, 9, 5240-5245; b) W. J. Li, Y. Y. Pan, R. Xiao, Q. M. Peng, S. T. Zhang, D. G. Ma, F. Li, F. Z. Shen, Y. H. Wang, B. Yang, Y. G. Ma, Adv Funct Mater 2014, 24, 16091614.

[6] C. Sutton, N. R. Tummala, D. Beljonne, J. L. Bredas, Chem Mater 2017, 29, 2777-2787.

[7] a) Z. Y. S. Li, * X. Xiao, H. Geng, K. Wang, X. Jin, Q. Liao, Y. Liao, Y Wu, J. Yao, and H. Fu*, Laser Photonics Review 2019, 13, 1900036; b) Z. Y. Yu, Y. S. Wu, L. Xiao, J. W. Chen, Q. Liao, J. N. Yao, H. B. Fu, J Am Chem Soc 2017, 139, 6376-6381.

[8] A. Lv, W. Ye, X. Jiang, N. Gan, H. Shi, W. Yao, H. Ma, Z. An, W. Huang, J Phys Chem Lett 2019, 10, 1037-1042.
[9] a) Goudappagouda, A. Manthanath, V. C. Wakchaure, K. C. Ranjeesh, T. Das, K. Vanka, T. Nakanishi, S. S. Babu, Angew Chem Int Edit 2019 58, 2284-2288; b) H. L. Ma, Q. Peng, Z. F. An, W. Huang, Z. G. Shuai, J Am Chem Soc 2019, 141, 1010-1015.

[10] A. J. Kuehne, M. C. Gather, Chem Rev 2016, 116, 12823-12864.

[11] T. Lu, F. W. Chen, J Comput Chem 2012, 33, 580-592.

[12] Q. Peng, D. Fan, R. Duan, Y. Yi, Y. Niu, D. Wang, Z. Shuai, The Journal of Physical Chemistry C 2017, 121, 13448-13456.

[13] Y. L. Niu, W. Q. Li, Q. Peng, H. Geng, Y. P. Yi, L. J. Wang, G. J. Nan, D. Wang, Z. G. Shuai, Mol Phys 2018, 116, 1078-1090.

[14] H. B. S. G. W. T. M. J. Frisch, G. E. Scuseria, M. A. Robb, J. R Cheeseman, G. Scalmani, V. Barone, B. Mennucci, G. A. Petersson, H Nakatsuji, M. Caricato, X. Li, H. P. Hratchian, A. F. Izmaylov, J. Bloino, G. Zheng, J. L. Sonnenberg, M. Hada, M. Ehara, K. Toyota, R. Fukuda J. Hasegawa, M. Ishida, T. Nakajima, Y. Honda, O. Kitao, H. Nakai, T. Vreven, J. A. Montgomery, Jr., J. E. Peralta, F. Ogliaro, M. Bearpark, J. J. Heyd, E. Brothers, K. N. Kudin, V. N. Staroverov, R. Kobayashi, J. Normand, K. Raghavachari, A. Rendell, J. C. Burant, S. S. lyengar, J. Tomasi, M. Cossi, N. Rega, J. M. Millam, M. Klene, J. E. Knox, J. B Cross, V. Bakken, C. Adamo, J. Jaramillo, R. Gomperts, R. E. Stratmann, O. Yazyev, A. J. Austin, R. Cammi, C. Pomelli, J. W. Ochterski, R. L. Martin, K. Morokuma, V. G. Zakrzewski, G. A. Voth, P. Salvador, J. J Dannenberg, S. Dapprich, A. D. Daniels, Ö. Farkas, J. B. Foresman, J. V. Ortiz, J. Cioslowski, D. J. Fox, Wallingford CT 2009

[15] X. Gao, S. M. Bai, D. Fazzi, T. Niehaus, M. Barbatti, W. Thiel, J Chem Theory Comput 2017, 13, 515-524.

[16] Q. Ou, G. D. Bellchambers, F. Furche, J. E. Subotnik, J Chem Phys 2015, 142. 


\section{Entry for the Table of Contents}

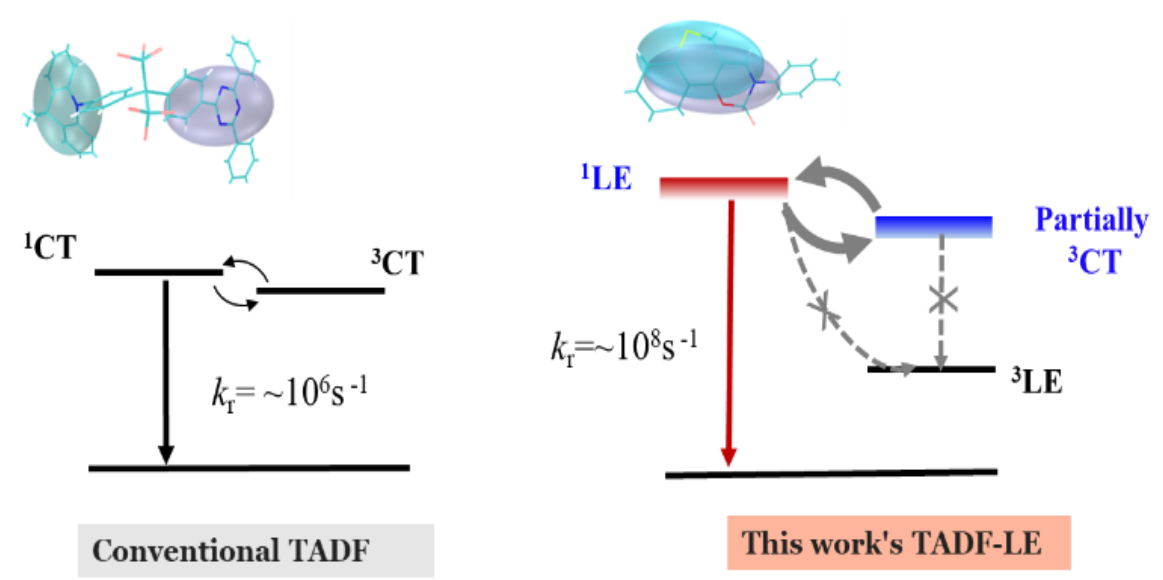

We provide a design strategy to realize TADF from a locally excited (LE) state through tuning the reverse intersystem crossing between the LE state and higher triplet states, based on which a boron difluoride derivative with TADF was theoretically designed and experimentally validated. This finding breaks through the traditional wisdom "the Charge-Transfer (CT) state is necessary prerequisite for TADF", and opens a new avenue for extending the TADF materials. 